\title{
Bisphosphonate-Conjugated Photo-Crosslinking Polyanionic Hyaluronic Acid Microbeads for Controlled BMP2 Delivery and Enhanced Bone Formation Efficacy
}

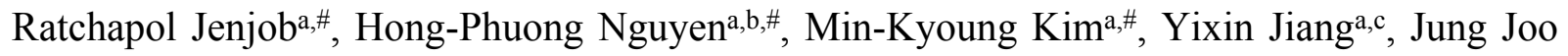
$\mathrm{Kim}^{\mathrm{a}}$, and Su-Geun Yanga,c*

aDepartment of Biomedical Science, BK21 FOUR program in Biomedical Science and Engineering, Inha University College of Medicine, Incheon 22212, South Korea

bInstitute of Research and Development, Duy Tan University, Da Nang 550000, Vietnam ${ }^{\mathrm{c} I n h a}$ Institute of Aerospace Medicine, Inha University College of Medicine, Incheon 22332, South Korea

\#These authors equally contributed to this work.

*Correspondence to Su-Geun Yang, PhD.

Department of Biomedical Science, Inha University College of Medicine, 366, Seohae-Daero, Jung-Gu, Incheon 22332, Republic of Korea.

Tel: +82-32-890-2832, Fax: +82-32-890-1199, E-mail: sugeun.yang@,inha.ac.kr

\section{Synthesis of methacrylated hyaluronic acid (MHA)}

HA was converted to HA-tetrabutylammonium salt (HA-TBA) by adding acidic ion exchange with Dowex 50WX8 resin and titrating with tetrabutylammonium hydroxide (TBA) until the $\mathrm{pH}$ of the mixture was neutral, followed by centrifugation and filtration to remove the resins, and then lyophilization (Yang, et al., 2011). Then, introduction of MA was performed in DMSO 
at $45^{\circ} \mathrm{C}$. Briefly, $1 \mathrm{~g}$ of HA-TBA powder was dissolved in $50 \mathrm{ml}$ of DMSO at $45^{\circ} \mathrm{C}$. Subsequently, $10 \mathrm{mg}$ DMAP and $3 \mathrm{ml}$ of MA were added into the reactor, then the reaction proceeded for $24 \mathrm{~h}$. After the reaction was completed, the product was purified by dialysis (25 $\mathrm{kDa} \mathrm{MWCO}$ ) against $150 \mathrm{mM} \mathrm{NaCl}$ for 2 days to remove TBA and distilled water for the final 2 days to remove DMSO, then the sample was lyophilized. Methacrylation of the sample was assessed with ${ }^{1} \mathrm{H}-\mathrm{NMR}$. The degree of methacrylation (\%) was approximately $44 \%$.

\section{Synthesis and characterization of HA-MA-alendronate (Alen-HMA) poymer}

MHA (0.5 g) was reacted with alendronate (Alen, $0.6 \mathrm{~g}$ ) in the presence of EDC/NHS system to obtain an alendronate-MHA (Alen-MHA) The reaction was carried out with $50^{\circ} \mathrm{C}$ for $24 \mathrm{~h}$. The product was purified using a dialysis machine with changing the distilled water several times for 12 h. Finally, the sample was lyophilized and the successful modification was confirmed by ${ }^{1} \mathrm{H}-$ and ${ }^{31} \mathrm{P}-\mathrm{NMR}$.

The functional group of MHA or Alen-MHA was confirmed by using ${ }^{1} \mathrm{H}-$ and ${ }^{31} \mathrm{P}-\mathrm{NMR}$ spectroscopy, respectively. The samples were dissolved in $\mathrm{D}_{2} \mathrm{O}$ and the NMR spectra were recorded at $25^{\circ} \mathrm{C}$ using a $500 \mathrm{MHz}$ NMR spectrometer. Degree of substitution (\% DS) of MA on HA was calculated by the ratio of the integral of MA protons at $\sim 5.5$ and $\sim 6.5 \mathrm{ppm}$ to that of HA at $2.1 \mathrm{ppm}$ (Yang, et al., 2011). The carbonyl $(\mathrm{C}=\mathrm{O})$ groups of MA after modification on HA backbone were analyzed by FTIR spectrometer (Wang, et al., 2003).

\section{Spinal bone fusion study on rat model}

The study was performed according to a previously reported method ${ }^{1,2}$. Sprague Dawley rats (Orient Bio Co., Seongnam, Korea) were assigned to the following two groups: BMP-2 alone 
and BMP-2/Alen-MHA MBs. Rats were anesthetized with continuous inhalation of $2 \%$ isoflurane. Two separate paramedic incisions were made in the lumbar fascia, $3 \mathrm{~mm}$ from the midline, after a posterior midline skin incision (Figure S5A). Then, the transverse process was exposed and decorated using low-speed burs (Seshin Co., Daegu, Korea). Subsequently, MBs were implanted on each side. Rats were housed in separate cages and allowed to eat and drink, and their condition was monitored daily. Spinal bone fusion was measured 8 weeks after treatment. Spinal bone fusion was quantified, and histochemistry was performed after the mice were euthanized. For the fusion quntification, score were manually evaluated as no fusion (0), partial fusion (1), or complete fusion (2), and the total sum scores were used to compare the fusion scores. For the histological assay, the dissected and formalin-fixed spines were demineralized with $50 \%$ formic acid and $10 \%$ sodium citrate, dehydrated in a graded ethanol series, and embedded in paraffin wax. Each specimen was fixed in 10\% formaldehyde solution, decalcified in formic acid for $48 \mathrm{~h}$, and embedded in paraffin. Serial cross-sections $(5 \mu \mathrm{m})$ were cut through the larger diameter of the defect and stained with HE and MT stain.

The mean fusion scores of the BMP-2/Alen-MHA MBs with manual palpation at 4 weeks after surgery showed a higher amount of fusion compared with those of the BMP-2 group (Figure S5C). MT staining also revealed that the cellular reactions indicative of bone formation were higher in the BMP-2/Alen-MHA MBs (Figure S5B). Ultimately, BMP-2/Alen-MHA MBs were able to heal spinal fusion more efficiently than BMP-2 alone did. 
A

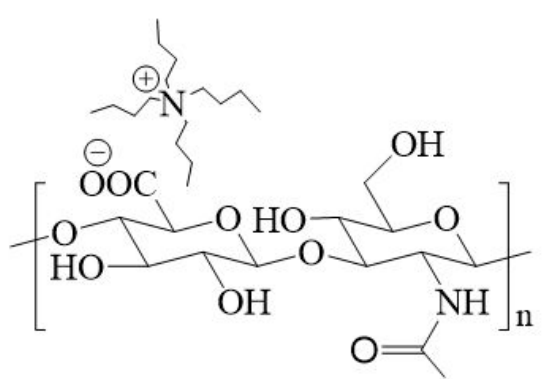

HA-TBA

B
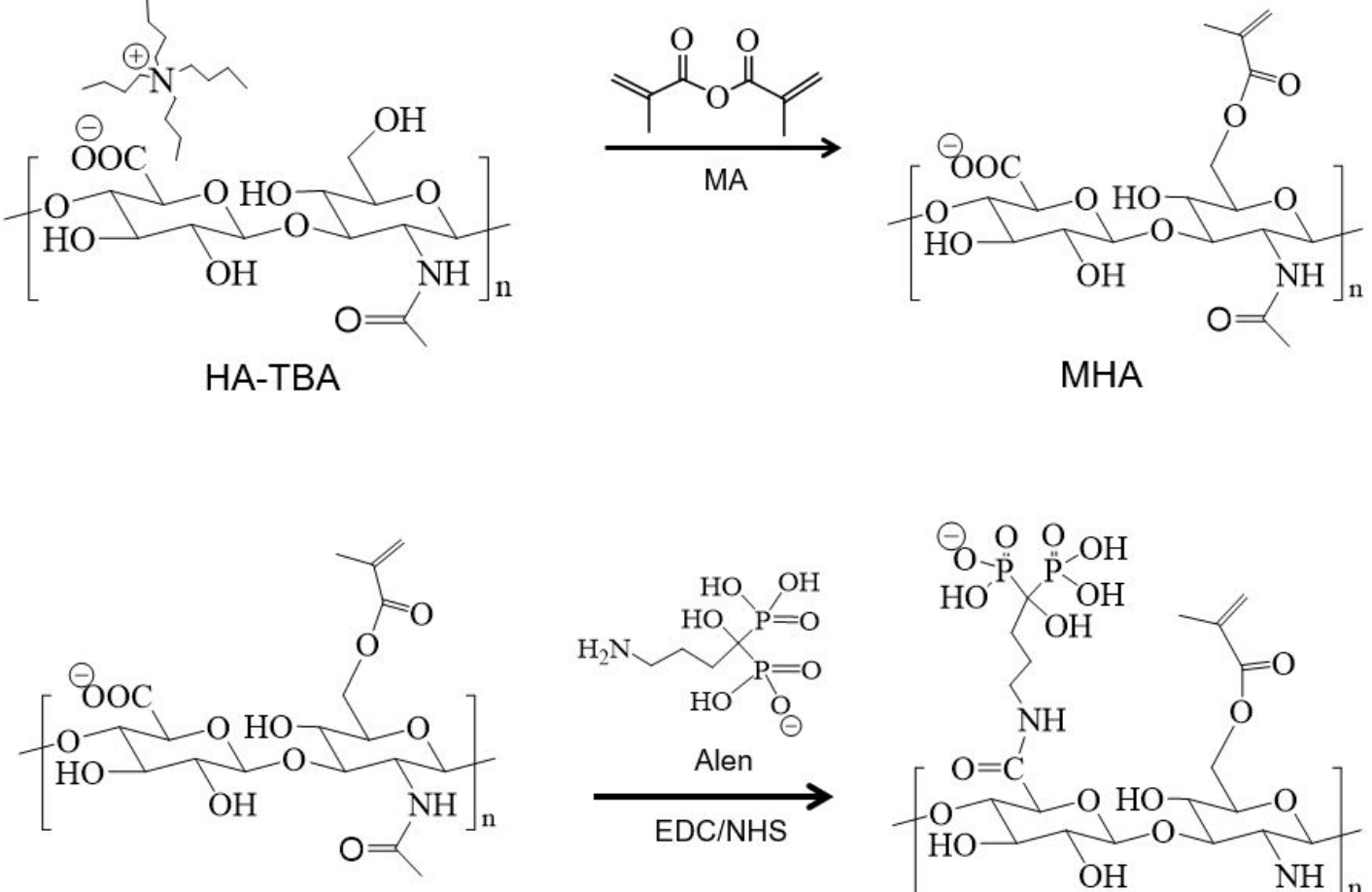

MHA
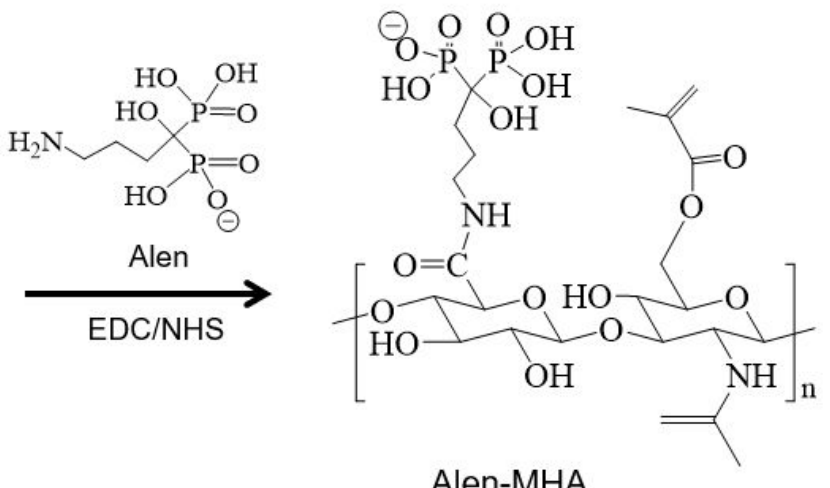

MHA

Alen-MHA

Figure S1. Schematic represent reaction of (A) MHA and (B) Alen-MHA 
$\mathbf{A}$

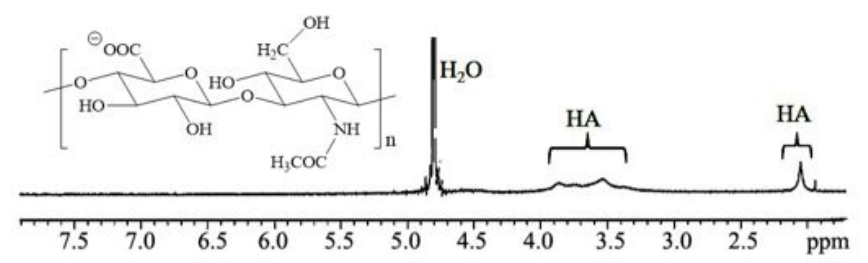

B

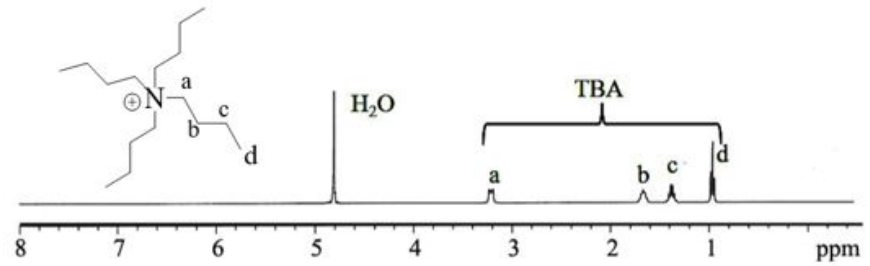

C
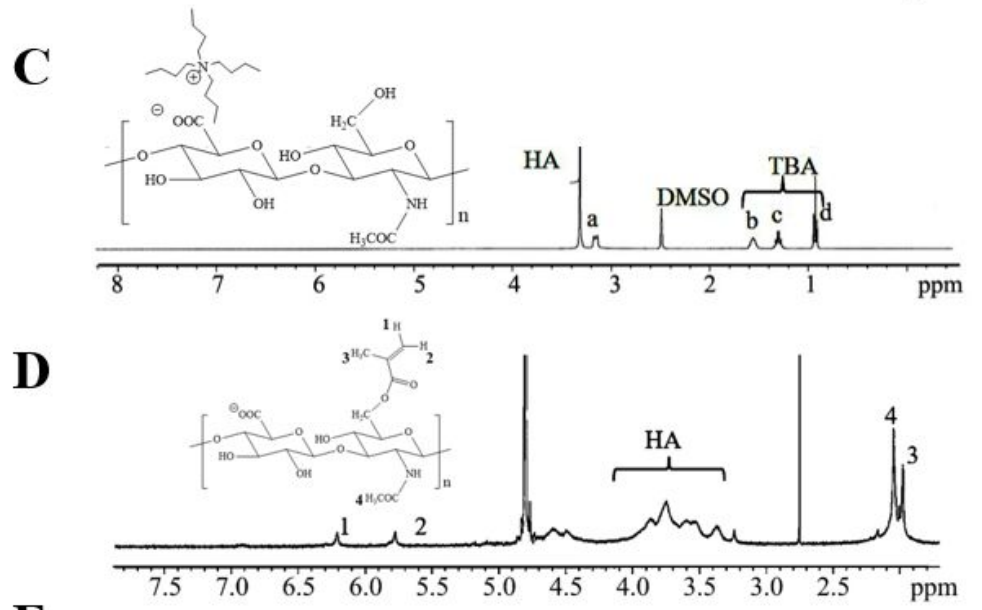

$\mathbf{E}$

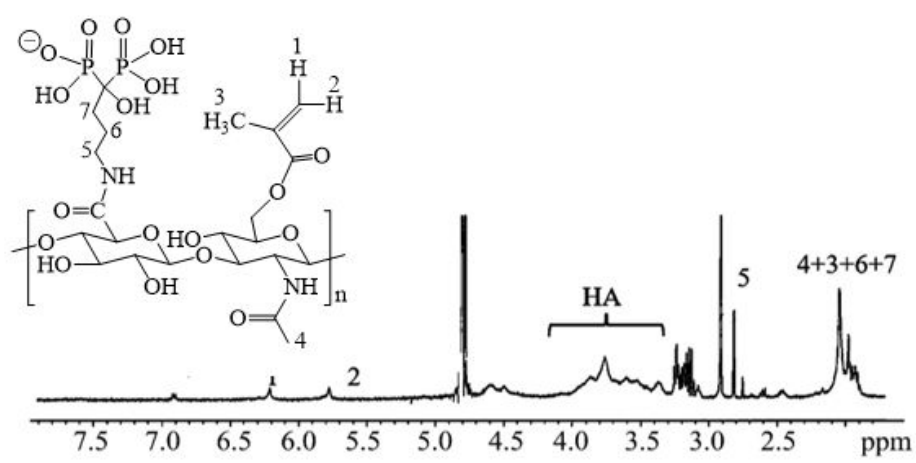

Figure S2. ${ }^{1} \mathrm{H}-\mathrm{NMR}$ spectra of (A) HA , (B) TBA, (C) HA-TBA, (D) MHA and (E) AlenMHA. 


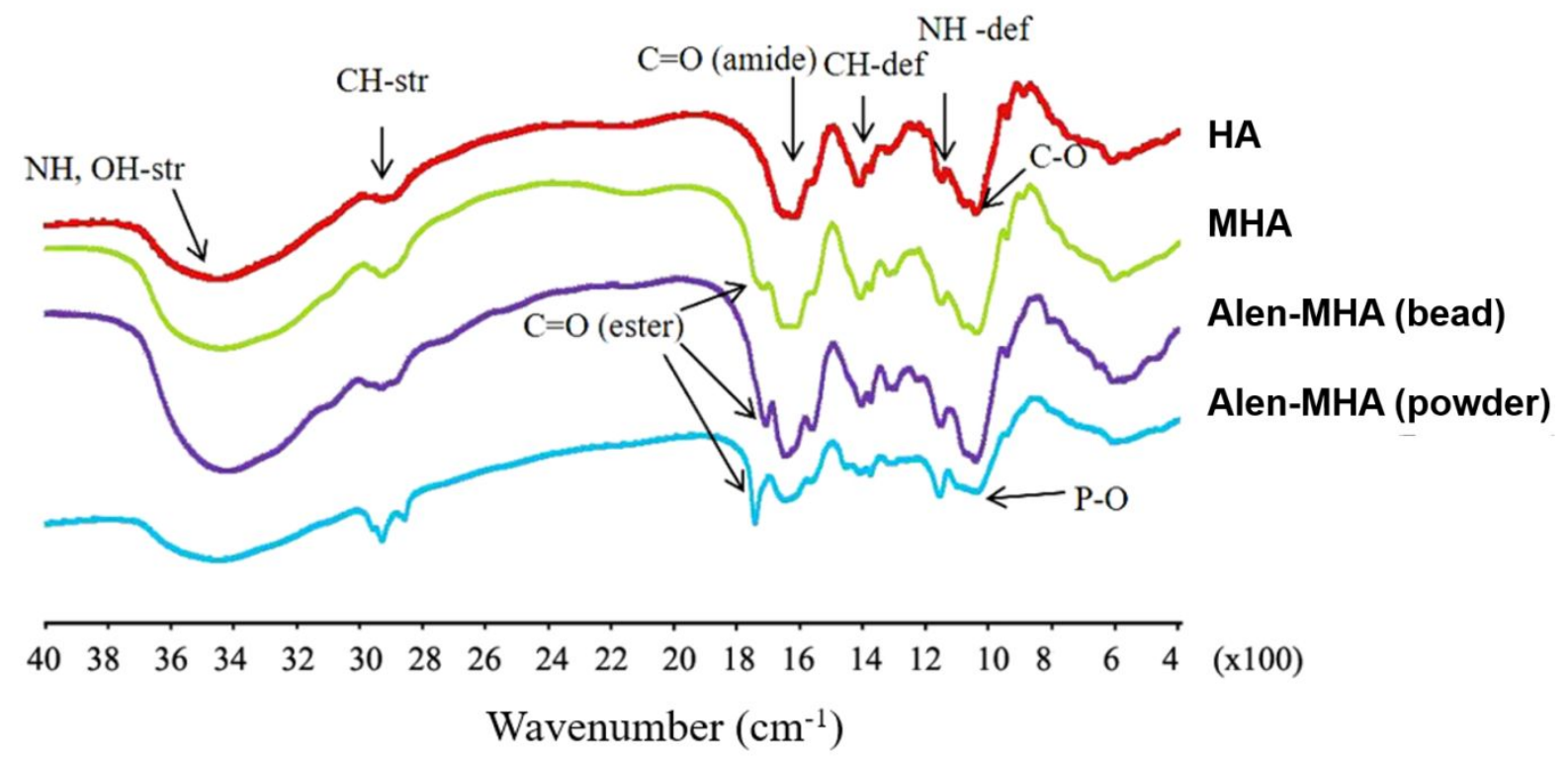

Figure S3. FTIR spectra of HA, MHA, Alen-MHA (bead), and Alen-MHA (powder). 
A

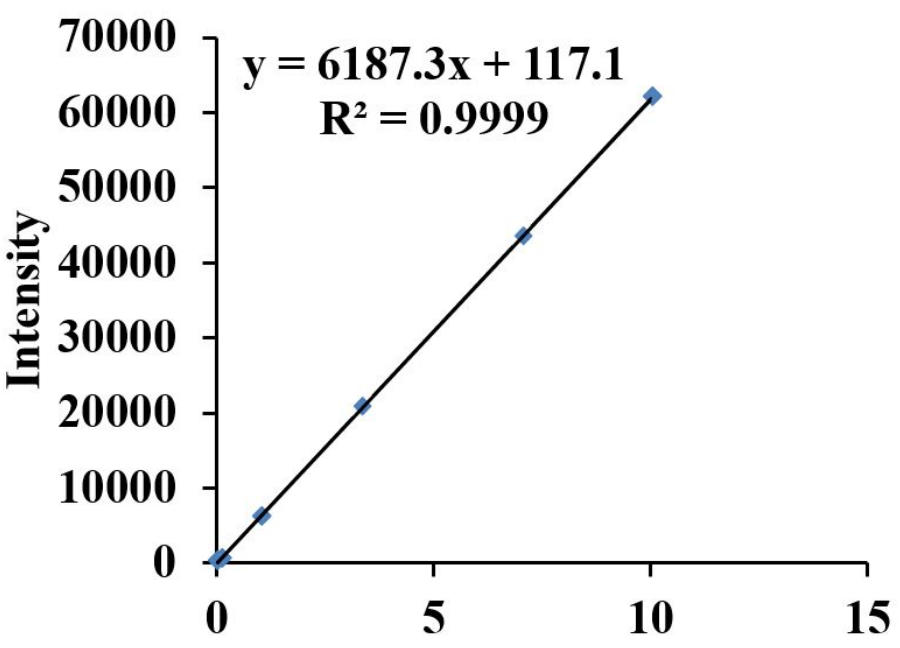

Phosphorus concentration (mg/L)

B

Percent (\%) phosphorus in Alen-MHA MBs

\begin{tabular}{lc}
\hline Sample name & Phosphorus (\%) \\
\hline Alendronate (Alen) & $22.69 \pm 2.57$ \\
Alen-MHA MBs & $2.40 \pm 0.21$ \\
\hline
\end{tabular}

Figure S4. ICP-OES assay of (A) calibration, (B) alendronate (Alen), Alen-MHA MBs for quantification of phosphorous content. 

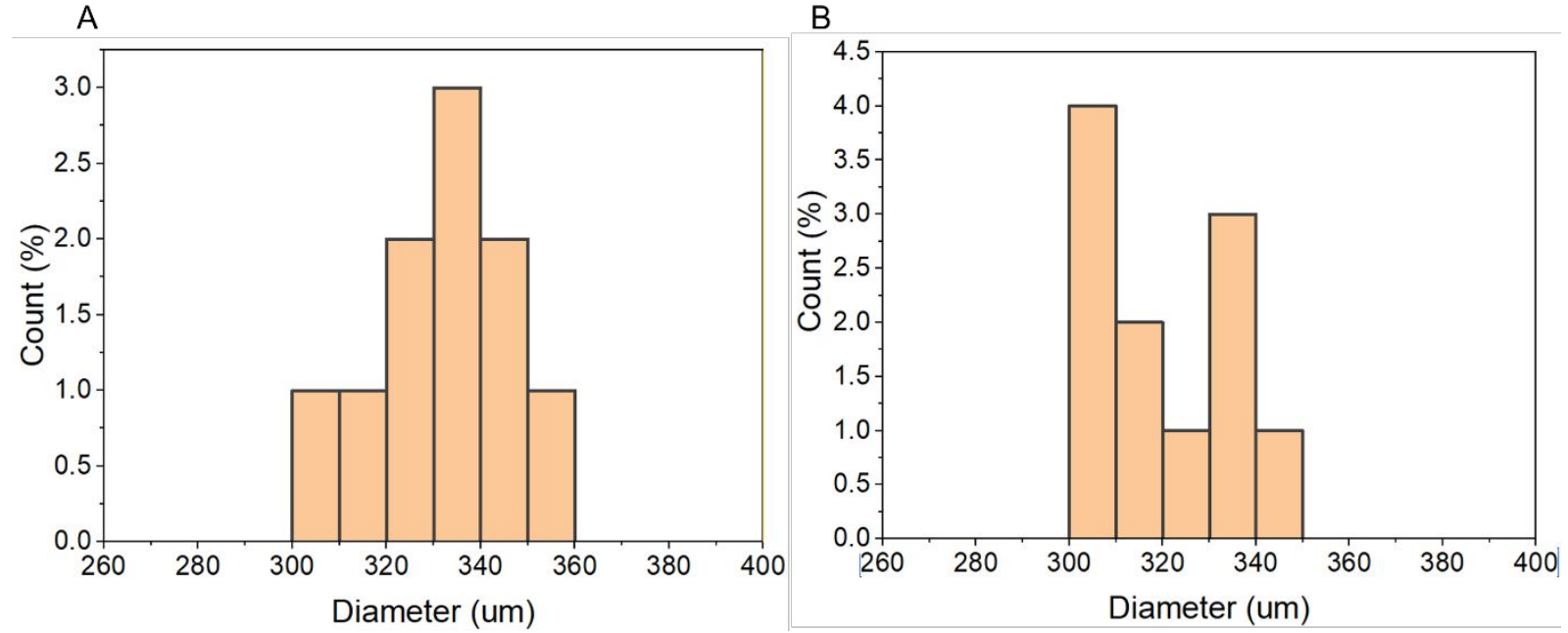

Figure S5. Size distribution of (A) MHA and (B) Alen-MHA. 


\section{A}
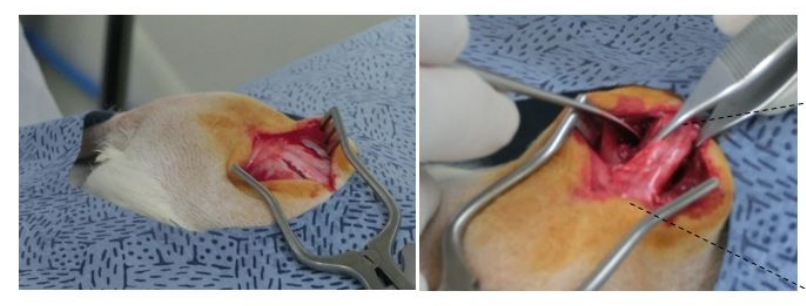

BMP2/Alen-MHA MBs

B

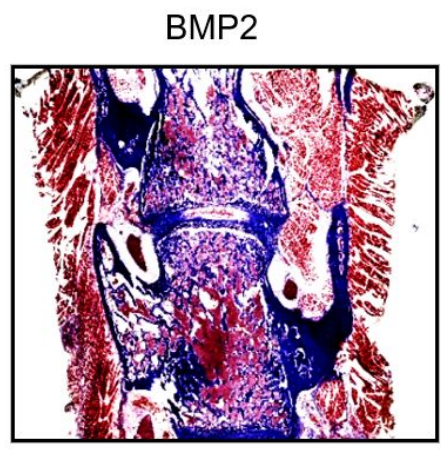

BMP2/Alen-MHA MBs

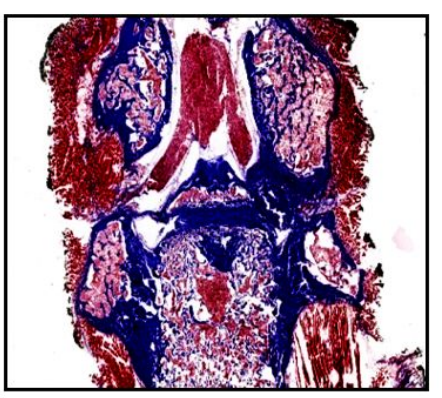

C

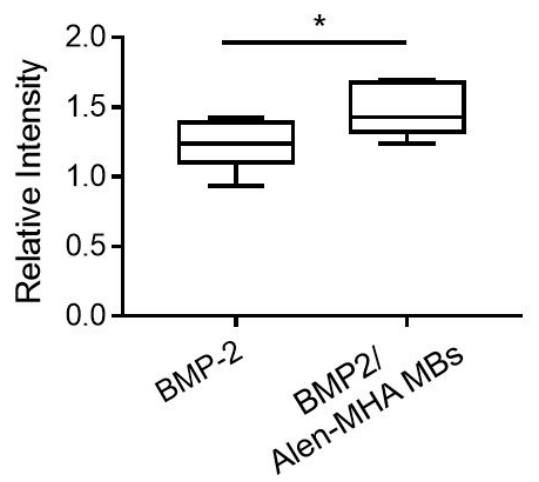

Figure S6. Rat spinal fusion efficacy of BMP-2/Alen-MHA MBs. (A) Masson trichrome staining of spinal after treatment of Alen-MHA MBs and BMP-2/Alen-MHA MBs. (B and C) Histology of spinal bone after fusion studies. Fusion scores were obtained using physical palpation (student t-test, $\mathrm{n}=3,{ }^{*} \mathrm{p}<0.1$ ).

1. Ryu, D.; Yoon, B. H.; Oh, C. H.; Kim, M. H.; Kim, J. Y.; Yoon, S. H.; Choe, S., Activin A/BMP2 Chimera (AB204) Exhibits Better Spinal Bone Fusion Properties than rhBMP2. $J$ Korean Neurosurg Soc 2018, 61, (6), 669-679.

2. Yoon, B. H.; Esquivies, L.; Ahn, C.; Gray, P. C.; Ye, S. K.; Kwiatkowski, W.; Choe, S., An activin A/BMP2 chimera, AB204, displays bone-healing properties superior to those of BMP2. J Bone Miner Res 2014, 29, (9), 1950-9. 